\title{
Medico-legal Management of Drug Abusers and Significance of Introducing an Effective Multidisciplinary
} Team

Konara Mudiyanselage Tikiri Bandara Gunathilake ${ }^{*} \otimes(D)$, Muditha Vidanapathirana ${ }^{2}$

${ }^{1}$ Office of the Judicial Medical Officer, Base Hospital, Awissawella, Sri Lanka

${ }^{2}$ Department of Forensic Medicine, Faculty of Medical Sciences, University of Sri Jayewardenepura, Sri Lanka.

\section{Introduction}

When drug misusers are produced before forensic practitioners, the police expects opinions on intoxication, fitness for an interview, drug searches, referral for rehabilitation etc. In Sri Lanka, according to "Drug-dependent persons (Treatment and Rehabilitation) Act, No. 54 of 2007", the forensic practitioners are expected to give their opinions with scientific reasoning. Therefore, when giving opinions on drug abusers, forensic practitioners face many practical problems.

\section{Objectives}

To evaluate the outcomes of medico-legal examinations of drug abusers presented to the Office of the Judicial Medical Officer, Provincial General Hospital, Ratnapura in 2017 and to develop an effective multi-disciplinary program to manage the same in Sri Lanka.

\section{Background history}

The objectives of the police were to obtain opinions on intoxication, addiction and refer to rehabilitation centres etc. Twenty-three drug misusers were produced for medico-legal examinations. Two denied drug misuse. In 19, the average time interval between arrest and producing was three days. In seven, there were no stigmata of abuse other than tattoos. One cannabis misuser had a psychiatric illness. Following examination and psychiatrist referral, the remaining 22 did not have evidence of acute intoxication or features of withdrawal syndrome. None of them was found to be drug-dependent and the referral for rehabilitation was not indicated.

\section{Conclusions}

Similar to the cases of child abuse, it is necessary to develop a multi-disciplinary follow-up system. A 'pre-arrest referral scheme' for drug misusers in the community and 'post-arrest referral scheme' when in custody. This can be further strengthened by adding a scientific test. Then the forensic practitioners will be in a better position to give opinions on drug dependency.

Keywords: Multi-disciplinary teams, Pre-arrest scheme, Post-arrest scheme

Copyright: @2018with the Medico-legal Journal of Sri Lanka.

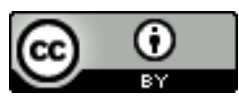

This is an open-access article distributed under the terms of the Creative Commons Attribution 4.0 International License, which permits unrestricted use, distribution and reproduction in any medium provided the original author and source are credited

Funding: None, Competing interests: None

Received: 17 April $2018 \quad$ Accepted revised version: 01 May 2018 Published: 30 June 2018

*Corresponding author: Gunathilake KMTB, Email: tikiriii_bandara@yahoo.com \4Tel: +94777705997 https://orcid.org/0000-0002-8485-4550

Cite this article as: Gunathilake KMTB, Vidanapathirana M. Medico-legal management of drug abusers and significance of introducing an effective multidisciplinary team. Medico-Legal Journal of Sri Lanka, 2018;6(1):22-26 DOI: http://dx.doi.org/10.4038/mljsl.v6i1.7368 


\section{Introduction}

Management of drug-related problems is a burden for any country irrespective of rich or poor or developing or developed. ${ }^{[1]}$ In Sri Lanka, drug abuse is a serious problem affecting young productive generations and creating social and legal problems due to increased crimes rates and it costs millions of rupees. ${ }^{[2]}$ In the United States of America, in 1999, it was 14 billion dollars. ${ }^{[3]}$ According to the US Surgeon Generals report on alcohol, drugs and health, 2015, the annual economic impact from the misuse of prescription drugs, illicit drugs or alcohol is $\$ 442$ billion. ${ }^{[4]}$

According to the 2004 executive summary of the United Nations office on drugs and crimes, there were about 45,000 regular users of heroin and about 600,000 users of cannabis in Sri Lanka. It was further estimated that between 1-2\% of heroin users are intravenous drug users (IDUs). ${ }^{[5]}$ In Sri Lanka, in 2015, from January to June, 8570 persons were arrested due to drug-related crimes. Of them, 1795 were heroin addicts, 5732 were married and $21 \%$ were between $30-34$ years of age. ${ }^{[6]}$ Further, when a married person becomes a drug addict there is a high chance of increasing gender-based violence and other related social problems. ${ }^{[6]}$ These show the gravity of the issue. Addicted persons have multiple drug-related problems other than intoxication. Further, they may have multiple medical problems directly or indirectly related to drug abuse. Forensic practitioners owe a duty of care to treat the acute problems and must act in good faith for the best interest of the patient. Moreover, they have legal and social obligations on public interest. However, they should act within the legal framework and they cannot deviate from the standard practice even when managing drug addicts. If there were legal provisions or protocols, those could have been used as guidelines and the forensic pathologists can safeguard themselves if they act accordingly and responsibly.

When the suspects of drug misusers are produced before forensic practitioners, the police may expect opinions on intoxication, fitness for an interview, drug searches (body packers, stuffers), referral for rehabilitation etc. ${ }^{[7]}$ In Sri Lanka, according to "Drugdependent persons (Treatment and Rehabilitation) Act, No. 54 of 2007 ", the forensic practitioners are expected give their opinions with scientific reasoning. ${ }^{[8]}$ Due to such expectations of the Act, when giving opinions on suspects of drug abuse, there are many practical problems for the forensic practitioners such as whether the suspects are dependents or not etc.

\section{Objectives}

To evaluate the outcomes of the medico-legal clinical examinations of drug abusers produced by the police to the Officer of the Judicial Medical Officer, Provincial General Hospital, Ratnapura, in the year 2017 and to develop an effective multi-disciplinary program to manage the drug abusers.

\section{Background history}

Twenty-three $(n=23)$ suspected drug misusers were produced for medico-legal clinical examination. The objectives of the police were to obtain opinions on intoxication, addiction and refer them to rehabilitation centres etc. All were males. Most $(n=20)$ were between 30-35 years of age. Two denied drug misuse. In 19 cases, the average time interval between arrest and producing was 3 days. In seven $(n=07)$, there were no stigmata of drug abuse other than tattoos on arms, which on the other hand may not be associated with drug use at all. One cannabis misuser had evidence of psychiatric illness. Following examination and psychiatrist referral, the remaining suspects $(n=22)$ did not have any evidence of acute intoxication or features of withdrawal syndrome. None of them was found to be drug-dependent and the referral for rehabilitation was not indicated.

\section{Discussion}

Substance misuse can lead to medical, social and legal problems. Not only the victim but also the society may be victimized due to drug abuse. Therefore, the use of substances would constitute a public health and social problem. ${ }^{[9]}$ According to the ICD-10 classification, the dependence syndrome is defined as being a cluster of physiological, behavioural, and cognitive phenomena in which the use of a substance or a class of substances takes on a much higher priority for a given individual than other behaviours that once had greater value. Therefore, "The Drug-dependent persons (Treatment and Rehabilitation) Act, No. 54 of 2007" expects the forensic practitioners to identify the drug-dependent and to refer to a rehabilitation programme.

There is a common belief among the legal authorities that the misuse and addiction are the same. The medico-legal examination may be complicated if the misuser is addicted to a drug. The seriousness of the addiction depends on the type of drug, state of intoxication and degree of addiction. Further, in addicts, the self and social harm are more. ${ }^{[9]}$

Once a drug misuser is produced before a forensic practitioner, a thorough clinical examination must be 
performed. Some of the general findings in substance misuse are neglect, poor nutritional state, dental caries, the external stigma of intravenous drug abuse, bizarre tattoos etc. ${ }^{[10]}$ In this case study, seven $(n=07)$ had tattoos but they were well-made ones rather than the bizarre types expected in intravenous drug addicts.

In intoxication, the misuser's central nervous system is affected by the abused drug. Clinical features depend on the type of drug used. Here, especially the level of consciousness, cognition, perception and judgment or behaviour etc are affected. ${ }^{[1]}$ Medicolegal examination of impairment assessment must be done to find out evidence of acute intoxication.

Only one person $(n=01)$ who was taking cannabis for a long time had features of a psychiatric illness. However, he did not have any clinical features of addiction or withdrawal. Other cases $(n=19)$ were produced before the forensic practitioner three days after the arrest and following medico-legal examination with referral to a psychiatrist, had no clinical features of acute intoxication or dependency. Withdrawal features of opioid addiction are severe in 48-72 hours and then gradually disappear. ${ }^{[9]}$

According to the Act No. 54/2007, when considering rehabilitation, most important factor is whether the person is a drug-dependent or not. Acute drug intoxication and drug addiction are completely different entities. According to ICD -10 classification, to diagnose drug dependency, 3 or more of following 7 features should persist for more than one year period. ${ }^{[12]}$

1. Some features can be identified by examination of the patient alone.

(i) Presence of tolerance, a need for an increased amount to achieve the desired effect or diminished effect with the same amount of drug.

(ii) Persistent desire and unsuccessful efforts to cut down or control substance use.

2. Some features could be identified by examining the victim and interviewing the family.

(iii) Taking substance in a larger amount for a longer duration.

(iv) The appearance of clinical features of withdrawal syndrome or taking the same or closely related substance to avoid withdrawal symptoms.

(v) A great deal of time is spent on activities necessary to obtain, use, or recover from its effects. (vi) Important social, occupational or recreational activities are given up or reduced because of substance use.

3. Some features can be identified from victim, family and the treating physicians.

(vii)Continue the substance abuse despite the knowledge of causing physical and psychological damage.

According to the above criteria, it is understood that for the diagnosis of drug addiction, most important factors are the history taken from the patient, family members and the treating physicians and the features should persist for more than one year. Further, according to the Act No. 54/2007, the forensic practitioners should give opinions with reasons and based on the facts. However, this could never be able to be achieved with present examination system, where the forensics practitioner meets only the patient which is also for too short a period.

Further, the suspects or even their relatives may not divulge reliable information. If the suspect is addicted, he will almost never consider rehabilitation. According to the above-mentioned definition of drug addiction, it is not possible for a medical professional, judicial medical officer or psychiatrist to decide whether the victim is a drug addict without a reliable history, long-term observation or monitoring of them because they get only a short contact time with drug misusers. Therefore, following examination and psychiatrist referral of above 23 suspects, all did not have dependent features. This highlights the value of community and custody based close monitoring system to identify and manage the drug-dependents. However, in Sri Lanka, in cases of child abuse, there is a special system to monitor the children and their families by the probation officers. Similarly, there should be a system to monitor the drug-dependents within their communities and custodies to facilitate and design their future medical management or rehabilitation.

In the United Kingdom, there is a program called 'drug referral or arrested referral scheme'. It is a partnership initiative set up to encourage substance misusers brought into contact with the police services to participate voluntarily in confidential help design to address their problems related to substance misuse. There are two approaches in 'arrested referral scheme'. (1) In information model, the police offer information in the form of a leaflet about drug and other relevant services to those they have arrested. However, the take-up rate of this model has been 
found to be low. (2) The proactive model involves 'drug workers' working in close cooperation with the police, often with the direct access to prisoners in custody. They may approach detainees in the police station themselves, providing an assessment either onsite or at a subsequent meeting. Here, information about drug services and encouragement to see advice is always provided by the drug workers. ${ }^{[11]}$

In Sri Lanka, we do not have such follow-up or monitoring system. We can adopt this system and appoint such 'drug officers' to develop a close rapport with drug misusers and their families. Further, the forensic practitioners can obtain necessary details from such officers to decide on the state of drug addiction of the suspects for legal purposes.

Therefore, we suggest the following multi-disciplinary scheme for Sri Lanka. (1) Pre-arrest referral scheme to monitor the addicts who are not indicated to arrest and are community-based. (2) Post-arrest referral scheme to be used in custody.

These 2 follow-up systems can decide whether to refer a person to an in-house rehabilitation centre or not. Multi-disciplinary teamwork among forensic practitioners, legal authorities, psychiatrist and other clinicians and 'Drug and alcohol officers' (DAO) can work together with the drug-dependent and can obtain valuable information to manage them and to formulate legal decisions on dependency. Such scheme will be helpful to the drug misuser and their families by reducing addiction and to the society by reducing the crime rate.

Moreover, co-morbid mental illness with drug dependence is a considerable problem. The fundamental principle that guides the treatment and care for these individuals, should be the integration of both specialist addiction services and mental health services. Addictions with severe mental illness can be handled by 'mental health services', while addiction with less severe mental disorders can be handled by 'specialist addiction services'.

As preventive strategies, there should be services at different levels. (1) There should be services offering drug-related information, advice, and referral to more specialist services. (2) There should be open-access harm-reduction interventions such as prevention of needle and syringe exchange. (3) There should be structured interventions provided by specialist community drug treatment services such as opioid stabilization programmes, opioid maintenance, reduction or detoxification programmes, structured social and psychosocial interventions such as counselling, couple therapy, etc. and (4) there should be residential rehabilitation interventions for those with severe co-morbid mental and/or physical health problems.

As indicated above, when the withdrawal syndrome is concerned, the medico-legal examination alone is not much useful. Moreover, the features of withdrawal syndrome may have been disappeared due to delayed presentation. Further, the best proof of long-term drug addiction is the hair analysis for drugs. ${ }^{[1]}$. By including such a test to our routine medico-legal examination, the forensic pathologist would be able to obtain scientific facts related to the sequence of drug usage and test the reliability of the history given by the suspects as well.

\section{Conclusions}

Drug misuse and addiction are completely different entities. Acute intoxication can be assessed by using the impairment assessments. However, to identify drug dependency, we highlight the value of community and custody based close monitoring systems.

According to the current system, it is almost impossible for the forensic practitioners to formulate an opinion regarding drug dependency without a reliable history and observations of the suspects. Therefore, similar to the cases of child abuse, it is necessary to develop a multi-disciplinary follow-up system, a 'pre-arrest referral scheme' for drug misusers in the community and 'post-arrest referral scheme' when in custody. This could be further strengthened by adding a scientific test. Then the forensic practitioners will be in a better position to give opinions on drug dependency.

\section{Referrals}

1. Perspective on the development dimension of drug control policy. UNDP. 2015. available from: https://www.unodc.org/pdf/technical_series_1 998-01-01_1.pdf

2. Hewawaduge S, Dorabawila S. Economic Consequences of Drug Abuse in Sri Lanka. Peradeniya Economics Research Symposium Department of Economics and Statistics, University of Peradeniya, Sri Lanka, 2015. pp 4550. available from:

https://arts.pdn.ac.lk/.../Economic\%20Consequen ces\%20of\%20Drug\%20Abuse

3. Stephens BG. Investigation of deaths from drug abuse. in Spitz and Fisher's Medico-legal 
Investigation of Deaths; $4^{\text {th }}$ ed. Illinois: Charles C Thomas;2006. p 1166.

4. US Surgeon General's report on alcohol, drugs and health, 2015. Available from: https://addiction.surgeongeneral.gov/executivesummary

5. Executive summary of the United Nations office on drugs and crimes, 2004. pp. 102-19. available from:

https://www.unodc.org/pdf/india/publications/so uth_Asia...Profile.../10_india.pdf

6. Statistical Report on Drug-related Arr 25 jri Lanka; Drug Abuse Monitoring System; vol-49, January-June; Research Division; National Dangerous Drug Board. Available in: http://www.nddb.gov.lk. Cited on 10.10.2017.

7. Shkrum MJ, Ramsay DA. Forensic Pathology of Trauma, common problems for the pathologist. $1^{\text {st }}$ ed. USA: Humana Press; 2007. p 428.
8. Drug-dependent persons (Treatment and Rehabilitation) Act, No. 54 of 2007

9. Lawrence B. Clinical Pharmacology. $7^{\text {th }}$ ed. London; Churchill Livingstone; 1995. p 318.

10. Karch SB. Drug abuse Handbook. $2^{\text {nd }}$ ed. Boca Raton: CRC Press; 2007.

11. Jason Payne JJ, Busuttil A, Smock W. Forensic Medicine: Clinical and Pathological Aspects. $1^{\text {st }}$ Ed. Amsterdam: Greenwich Publication; 2003. pp 518-9 and 618.

12. International Code of Disease ClassificationICD-10. Available in:

http://www.who.int/ classifications/icd/. Cited on 10.10.2017 